\title{
Review on Campylobacteriosis in Ethiopia Perspective
}

\author{
Kula Jilo ${ }^{1, ~ *, ~ W a k t o l e ~ Y a d e t a ~}{ }^{2}$, Gudata Kanchu ${ }^{3}$ \\ ${ }^{1}$ Veterinary Epidemiology, Addis Ababa University, College of Agriculture and Veterinary Medicine, Bishoftu, Ethiopia \\ ${ }^{2}$ Veterinary Clinical Medicine, Jimma University, College of Agriculture and Veterinary Medicine, Jimma, Ethiopia \\ ${ }^{3}$ Teltelle District Livestock Resource Development Office, Teltelle, Ethiopia
}

Email address:

kula.jilo1@gmail.com (K. Jilo)

${ }^{*}$ Corresponding author

\section{To cite this article:}

Kula Jilo, Waktole Yadeta, Gudata Kanchu. Review on Campylobacteriosis in Ethiopia Perspective. World Journal of Public Health. Vol. 6, No. 2, 2021, pp. 25-30. doi: 10.11648/j.wjph.20210602.11

Received: December 7, 2020; Accepted: March 25, 2021; Published: April 7, 2021

\begin{abstract}
Campylobacteriosis is widespread worldwide infecting all warm blooded animals including human beings. Campylobacter species are a leading cause of bacterial-derived foodborne disease Campylobacter is a gram-negative comma shaped rods, microaerophilic and motile. Campylobacter jejuni and Campylobacter coli are the major cause of enteritis in human being and mainly transmitted to humans via handling and eating raw or undercooked meat, especially poultry. It causes watery diarrhea, abdominal pain, vomiting, fever, and nausea with nervous system complications in humans. Warm-blooded farm animals such as poultry, pigs, cattle and sheep are major reservoirs for Campylobacter species. Most Campylobacter infections are acquired by consuming or handling poultry, the ideal way to control the number of human infections would be to limit contamination of poultry and its products at different levels. Routine use of antibiotic prophylaxis to prevent Campylobacter infections is not recommended. In immune-competent individuals, Campylobacter enterocolitis self-limited, with mild to moderate symptoms but supportive care with oral rehydration is the preferred treatment. In Ethiopia its highly prevalent ranging from 8-13.7 in human and 9.4-72.7 in food animals. High prevalence of zoonotic Campylobacter species has been isolated from animal products and animal feaces. Therefore, consumption of raw meat, unpasteurized milk and untreated water should be avoided. Public awareness creation to minimize risk of campylobacteriosis is also very important.
\end{abstract}

Keywords: Campylobacter, C. jujeni, C. coli, Ethiopia

\section{Introduction}

Campylobacter species are a leading cause of bacterialderived foodborne disease worldwide [1]. Campylobacter is a gram-negative corkscrew shaped rods and flagellated motile [2]. Among 28 Campylobacter species C. jejuni and C. coli are the major cause of enteritis in human [3]. Campylobacter are mainly transmitted to humans via handling and consumptions of raw meat and cause watery diarrhea, abdominal pain, vomiting, fever, and nausea with nervous system complications. Farm animals like poultry, pigs, cattle and sheep are major reservoirs for campylobacter species. It is mainly asymptomatic; however abortions, enteritis and infertility can occur in various species of animals $[4,5]$.

An ideal way to control the number of human infections would be to limit contamination of poultry and its products at different levels. Routine use of antibiotic prophylaxis to prevent Campylobacter infections is not as such satisfactory. Campylobacter enterocolitis is generally self-limited in most people [6]. In Ethiopia its highly prevalent ranging from 813.7 in human and 9.4-72.7 in food animals [7]. Due to its devastating public health threat international organizations like WHO, FAO and OIE are collaboratively working on Campylobacteriosis to address health risks at the humananimal-ecosystems interface [1].

\section{History}

It was the symptom that was described by Escherich not the species. The Campylobacter species were described (as Vibrio) for the first time 1913 by McFadyean and Stockman as a cause of bovine and ovine infertility and abortion. The 
first time Campylobacter spp. were isolated from humans was in conjunction with a milk-borne outbreak1938. In the late 1950s, Campylobacter spp. was isolated from blood samples of children with diarrhoea. Crucial step was taken in Belgium in the early 1970s when Campylobacter were isolated from human faeces [2]. Until, 1970s Campylobacter species were associated with other human infections before it were fully recognized as human pathogens. In 1968, it was isolated from human faeces and later in mid-1900s; Campylobacter species were also isolated from animals that developed abortion and sterility. Due to it is spiral rod shaped bacteria the name Campylobacter is derived from two Greece word 'campylos' meaning curved and 'baktron' meaning rod $[2,8]$.

\section{Etiology}

The genus Campylobacter consists of 28 species and 8 sub-species. C. jejuni is the major frequently reported Campylobacter species ( $80 \%$ to $90 \%$ ) followed by C. coli (5\% to 10\%)[9]. Campylobacter are Gram-negative corkscrew shaped rods, motile with a width of 0.2-0.8 $\mu \mathrm{m}$ and a length of 0.5-5 $\mu \mathrm{m}$. Some Campylobacter species are thermotolerant and grow at temperatures between $37^{\circ} \mathrm{C}$ and $42^{\circ} \mathrm{C}$, with C. coli and C. jejuni having an optimal growth temperature of $42^{\circ} \mathrm{C}$ and $\mathrm{pH}$ 6.5-7.5. The majority of Campylobacter species are microaerophilic and need reduced oxygen (3-10\%) and raised CO2 (5-10\%) levels. Campylobacter is sensitive to freezing and drying but it is death rate is dependent on temperature. They can survive at refrigeration temperatures $\left(4^{\circ} \mathrm{C}\right)$ and in meat stored frozen (at $-18^{\circ} \mathrm{C}$ to $-22^{\circ} \mathrm{C}$ ) for several weeks [10].

\section{Epidemiology}

Campylobacteriosis is widespread worldwide colonizing all warm blooded animals including human beings [4]. Campylobacter species are a part of the gut flora in many domestic and wild animals. Warm-blooded farm animals such as poultry, pigs, cattle and sheep are major reservoirs and source infection for human being. Campylobacter have also been found among wild birds, wild animals and even in non-vertebrate vectors such as flies. The majority of human infections are caused by C. jejuni (80-90\%) and C. coli (5$10 \%$ ). C. jejuni is the most prevalent Campylobacter found in most animals, with the exception for pigs for which $C$. coli [9].

There is quite epidemiological difference between low, middle and high income countries which likely arise from differences in diagnostic techniques, biocontrol protocols, food practices, nutritional status, environmental hygiene, climatic condition and the abundance of natural reservoirs $[10,11]$. In low and middle income countries, Campylobacter infections are hyperendemic among young children aged less than 2 years with up to 2 episodes per child and commonly asymptomatic in both children and adults [12]. In high income countries, asymptomatic
Campylobacter infections are unusual but the average number of Campylobacter infections is less than one per lifetime [12]. Campylobacter infection is usually more prevalent to children suggesting that early life exposure might elicit the development of protective immunity however subsequent asymptomatic shedding are observed [13].

Poultry is a leading source of food-related Campylobacter species to humans responsible for outbreaks are consumption of poultry products [11]. Huge amount of Campylobacters are excreted in poultry feaces and contamination during slaughter contaminate the surrounding environment as the crop of poultry is an important niche for Campylobacter species. Once colonized, chickens remain colonized until they are slaughtered and thus, chicken feces are also a potential source of transmission to the environment or humans when poultry manure is used as a fertilizer [14]. Thus, abundance of poultry farms in a given area is one of the epidemiological determinants of campylobacter infections due to soil, water, dust, air and fomites contaminations [15].

Campylobacter infection is often endemic in areas with untreated drinking-water, poor sanitation and close contact with animals and environmental sources [16]. Poultry production in a free range system has a much greater risk of infection compared to conventional production and increased the difficulties in control [17]. Direct contact with farm animals and consumptions of raw farm animal products play a significant role in epidemiology of campylobacteriosis. Unpasteurized milk source from dairy cattle has also been implicated in a number of campylobacteriosis outbreaks [10]. Wild animals are potential reservoirs of Campylobacter species [9]. Among all the host species studied, wild birds are most likely to carry Campylobacter species. Some species of wild birds are observed as carrier but their role in Campylobacter transmission to human is not determined yet [18]. In general, age, farming system, sanitary measures in farms, immunodeficiency, exotic breed, food contamination, animal contact, international travel, and abundance of reservoir hosts are main determinant factors [4].

\section{Source Infection and Transmission}

Campylobacter are mainly transmitted to humans via handling and eating raw or undercooked meat, especially poultry [12]. Consumption unpasteurized milk, contaminated water and food, person to-person transmissions through fecal-oral or fomites are also potential source of transmission for Campylobacter infections [4, 15]. The usual route of Campylobacter transmission in animals is horizontal transmission through ingestions of Campylobacter with contaminated water, feed, soil and fomites (Little et al., 2010). Vertical transmission from an infected bird to its offspring prior to shell formation is rare, although "pseudovertical transmission" from parent flocks to their chickens via fecal contamination of shells can occur [3]. C. fetus subspecies venerealis transmitted venereally in cattle [20]. 


\section{Clinical Signs}

\subsection{In Animals}

Campylobacter species are common colonizers of the gut flora of warm-blooded animals including livestock, domestic pets, and wild animals, and especially prevalent in avian species [21]. Campylobacter species can cause abortions, enteritis, and infertility in various species of animals. In chicken C.jejuni infection lead to prolonged inflammation, damage of the gut, and diarrhea [22]. C. jejuni and occasionally $C$. coli cause enteritis in cats, sheep, dogs, poultry, and calves some species of laboratory animals with more severity in young animals. Mucoid diarrhea with flecks of blood with or without fever is typical in calves. In sheep $C$. fetus subsp. fetus and C. jejuni cause stillbirth, abortion, weak lambs, reduction in milk production, prolonged lambing with immunity revival after re-infection [23].

\subsection{In Human}

Most of the human Campylobacter enteritis is caused by $C$. jejuni and $C$. coli manifested as gastrointestinal and extra intestinal clinical signs [4]. Gastrointestinal illness is an acute and self-limited characterized by mild watery offensivesmelling to severe bloody diarrhea, vomiting, nausea, fever. Extraintestinal manifestations of Campylobacter infection include meningitis, endocarditis, septic arthritis, osteomyelitis, and neonatal sepsis. The most important postinfectious complication of $C$. jejuni infection is the Guillain-Barré syndrome (GBS) an acute demyelinating disease of the peripheral nervous system which cause flaccid paralysis [24].

\section{Diagnosis}

\subsection{Culture and Isolation}

Campylobacteriosis can be diagnosis by direct examination of feces and isolation of the organisms confirmed by culture of the organism [5]. Campylobacter species require a microaerobic atmosphere containing about $5 \%$ oxygen, $10 \%$ carbon dioxide, and $85 \%$ nitrogen. The conventional method for isolating the common enteric Campylobacter species (C. jejuni and C. coli) from faeces is primary plating on selective media and incubation at $42{ }^{\circ} \mathrm{C}$ for 48 to 72 hours in a microaerobic atmosphere with commercially available anaerobic jars. Selective media blood based, or blood-free charcoal-based, and contain one or more antibiotics, can be used. Suspect colonies should be screened with oxidase test, wet mount preparation under dark-field or phase-contrast microscope, and Gram stain. Isolation of Campylobacter spp. using enrichment culture is time consuming and complex [25]. Reducing the time taken to confirm the presence or absence of Campylobacter spp. Rapid techniques such as real-time PCR can detect campylobacters from complex samples but blood in enrichment culture can inhibit the PCR reaction, if applied directly to enriched samples [25].

\subsection{Identification}

Identification of Campylobacter species requires tests including morphological appearance, biochemical reactions, growth temperature, tolerance tests and antibiotic sensitivity to cephalothin and nalidixic acid. Polymerase chain reaction (PCR) can be applied in the diagnosis of campylobacteriosis, using either species-specific or multiplex reactions based on ribosomal $16 \mathrm{~S}$ gene sequences, and microarray-based identification tests, have been developed [5].

\section{Prevention and Control}

Most Campylobacter infections are acquired by consuming or handling poultry, the ideal way to control the number of human infections would be to limit contamination of poultry flocks and its products at different levels. Prevention of many outbreaks of $C$. jejuni infection could be accomplished with avoidance of the consumption of unpasteurized milk; this should be emphasized to pregnant women, the elderly, immunocompromised persons, persons who travel to low or middle income countries and campers should be cautioned against drinking untreated water [1].

\subsection{At Farm Level}

Campilobacteriosis is common in domestic and wild animals and therefore in the environment. For this reason, it is important to reduce contamination of chicken rearing houses from such sources. Use of hygiene barriers at the entrance to poultry houses, controlling the entry of farm personnel, the provision of hand-washing facilities, the performance of boot dips, and the use of house-specific boots and over shoes have all been shown to be effective [22]. Segregation of Campylobacter-positive flocks from negative flocks at the slaughter house, and slaughtering of the positive flocks an effective method of reducing spread of contamination. Disinfection measures of their food and water, treatment of their manure, and isolation of the contagiously ill. Emptying and cleaning water troughs more regularly have been shown to reduce the risk of colonization of cattle by Campylobacter [1].

\subsection{At the Food Animals Processing Level}

The intestinal tracts of chickens contains huge amount of campylobacter potential contaminant of the slaughter house, environment and their food products. At this point, treatment with organic acids, UV light, chemical dip tanks for carcasses help to reduce the number of Campylobacter organisms [26]. Scalding, freezing or irradiating reduces bacterial numbers by killing the pathogens and limiting the level of crosscontamination in the kitchen [26].

\subsection{At Home Level}

Safe food-preparation and appropriate consumption habits is important in the prevention of infections of Campylobacter species [27]. Adequately cooking and the use of a meat 
thermometer help to ensure that temperatures adequate to kill Campylobacter species organisms are achieved. Cutting boards and utensils used in handling uncooked poultry or other meats should be washed with hot soapy water before being used for preparation other foods that are eaten raw. Separation of ready to eat, raw food and hand washing should be applied. Persons with any acute diarrheal illness should avoid preparation and handling of food until their illness resolves. Avoidance of eating raw meat, unpasteurized dairy products, and exposure to animals such as pet animal with diarrhea should be avoided. All people, especially those who handle pets or other animals, should wash their hands before eating [26, 27].

\section{The Status of Campylobacters in Ethiopia}

In Ethiopia, there are only few reports on the prevalence of Campylobacters isolated from humans and food animals [28, 29]. However, prevalence ranging from 8-13.7 in human and 9.4-72.7 in food animals have been reported (Table 1). The first study of human campylobacter prevalence in Ethiopia done in 1992 at diarrhoeic patients at Tikur Anbessa hospital reported that prevalence of 13.7 Campylobacters from total of 630 examined patients [30]. Similarly, study conducted at Jimma on 430 children who had diarrhoea reported prevalence of Campylobacter $11.6 \%$ from stool specimen [28].

A study conducted in urban and rural farm animal in Jimma in 2004 on 485 various food animals (cattle, poultry, pigs and sheep) to isolate Campylobacter species were isolated $192(39.6 \%)$ from fecal specimens. The highest isolation rate was recorded among chickens (68.1\%), followed by pigs $(50.0 \%)$, sheep $(38.0 \%)$ and cattle $(12.7 \%)$. Among these 192 Campylobacter species isolated, 135
(70.3\%) were identified to be $C$. jejuni, $51(26.6 \%)$ were $C$. coli and $6(3.1 \%)$ were $C$. lari [29]. Similarly, another survey done in Addis Ababa and in and around Bishoftu in 2007 on 540 raw meat samples from different food animals isolated Campylobacter species from 50 (9.35\%) samples. The highest prevalence was reported in chicken $(21.7 \%)$ followed by sheep meat $(10.5 \%)$, pork meat $(8.5)$ goat meat (7.6\%) and beef (6.25). Among the 50 isolates of Campylobacter species $78 \%$ were $C$. jejuni, $18 \%$ and $4 \%$. coli [31] (Table 1).

A cross-sectional study conducted on apparently healthy sheep and goat slaughtered at a private export abattoir in Bishoftu in 2008 reported $40(10.1 \%)$ prevalence of Campylobacter species out of 398 carcasses examined, of the 40 thermotolerant Campylobacter isolates, $C$. jejuni and $C$. coli accounted for $29(72.5 \%)$ and $11(27.5 \%)$, respectively [32]. On other hand, Campylobacter survey on 220 chickens and 210 humans in Bahir Dar in 2008 revealed prevalence of thermophilic Campylobacters of $8 \%$ and $72.7 \%$ in humans and chickens respectively. In humans, $94.1 \%$ of the isolates were $C$. jejuni and $5.9 \%$ were $C$. coli. $C$. jejuni was a predominant species of thermophilic Campylobacters [33]. Study undertaken at Debre Birhan in 2012 on 70 fecal and 310 carcass samples found that $33(10.6 \%)$ with $87.9 \% C$. jejuni and $12.1 \%$ C. coli from fecal samples whereas, 15 (21.4\%) Campylobacter isolates were found from 70 carcass samples from which $C$. jejuni and $C$. coli accounted for 93.3 and $6.7 \%$ respectively [34]. A cross sectional study conducted on 384 raw bovine meat samples in Addis Ababa in 2013 isolated in 36 (9.4\%) Campylobacter species that showed that 28 (78\%) C. jejuni and C. coli 8 (22\%) [7]. In, Gambella, Out of 368 fecal samples taken from farm animals, overall prevalence of $56.5 \%$ with $83.7 \%$ C. jejuni, $12.9 \% C$. coli and 3.4\% C. lari was reported [35].

Table 1. Status of campylobacter in Ethiopia.

\begin{tabular}{|c|c|c|c|c|c|c|}
\hline Area & Origin of sample & Prevalence & C.jejuni & C.coli & C.lari & References \\
\hline Tikur Ambesa H & Human & 13.7 & - & - & - & {$[30]$} \\
\hline Jimma & Human & 11.6 & - & - & - & {$[28]$} \\
\hline Jimma & Food animal & 39.6 & $70.3 \%$ & $26.6 \%$ & $3.1 \%$ & [29] \\
\hline Bishoftu & Food animal & 9.35 & $78 \%$ & $18 \%$ & $4 \%$ & {$[31]$} \\
\hline Bishoftu & Sheep and goat & 10.1 & $72.5 \%$ & $27.5 \%$ & - & {$[32]$} \\
\hline Bahir-dar & Human & 8 & $94.1 \%$ & $5.9 \%$ & - & {$[33]$} \\
\hline Debre-birhan & Sheep & 15.7 & $90.6 \%$ & $9.4 \%$ & - & {$[34]$} \\
\hline Addis Ababa & Cattle & 9.4 & $78 \%$ & $22 \%$ & - & [7] \\
\hline Gambella & Food animal & 56.5 & $83.7 \%$ & $12.9 \%$ & $3.4 \%$ & {$[35]$} \\
\hline
\end{tabular}

\section{Conclusion and Recommendations}

Attempts of studies on Campylobacter in Ethiopia are encouraging. High prevalence of Campylobacter infections have been reported from different parts of Ethiopia both in human and animals. High prevalence of zoonotic Campylobacter species (C.jejuni and C.coli) has been also isolated from animal products and animal feaces. However, more surveillance in covering wider areas in collaboration with international organizations and stake holders is highly recommended. Consumption of raw meat, unpasteurized milk and untreated water should be avoided, Public awareness creation to minimize risk of campylobacteriosis is also very important. 


\section{References}

[1] World Health Organization. (2015): WHO's first ever global estimates of foodborne diseases find children under 5 account for almost one third of deaths. News release, Geneva, Switzerland.

[2] Butzler, JP (2004): Campylobacter, from obscurity to celebrity. Clin Microbiol Infect Off Publ Eur Soc Clin Microbiol Infect Dis. 10 (10): 868-76.

[3] Centers for Disease Control and Prevention (2019): Reports of Selected Campylobacter Outbreak Investigation, Atlanta USA.

[4] Centers for Disease Control and Prevention (2013): Recurrent outbreak of Campylobacter jejuni infections associated with a raw milk dairy - Pennsylvania, April-May 2013. Morb Mortal Wkly Rep 62: 702.

[5] Man SM, Kaakoush NO, Octavia S, Mitchell H. (2010): The internal transcribed spacer region and ewe tool for use in species differentiation and delineation of systematic relationships within the Campylobacter genus. Appl Environ Microbiol 76: 3071-3081.

[6] Masci, JR., Wormser, GP. (2005): Mandell, Douglas, and Bennett's Principles and Practice of Infectious Diseases, 6th Edition Edited by Gerald L. Mandell, John E. Bennett, Raphael Dolin Philadelphia: Elsevier Churchill Livingstone, Clinical Infectious Diseases 41: 277.

[7] Faris G (2015): Identification of Campylobacter spp. and their Antibiotic resistance pattern from raw bovine meat in Addis Ababa, Ethiopia. Int J Microbial Immu Res 4: 001-005.

[8] Skirrow, MB and John, Mc (2006): Fadyean and the centenary of the first isolation of Campylobacter species. Clin Infect Dis off Publ Infect Dis Soc Am. 43 (9): 1213-17.

[9] Levallois P, Chevalier P, Gingras S, Dery P, Payment P, Michel P, Rodriguez M. (2014):. Risk of infectious gastroenteritis in young children living in Quebec rural areas with intensive animal farming: results of a case-control study (2004-2007). Zoonoses Public Health 61: 28-38.

[10] Wagenaar JA, French NP, Havelaar AH. (2013): Preventing Campylobacter at the source: why is it so difficult? Clin Infect Dis 57: $1600-1606$.

[11] Kaakoush, N. O., Castaño-Rodríguez, N., Mitchell, H. M., \& Man, S. M. (2015). Global epidemiology of Campylobacter infection. Clinical microbiology reviews, 28 (3), 687- 720.

[12] Mullner, P., Shadbolt, T., Collins-Emerson, J. M., Midwinter, A. C., Spencer, S. E. F., Marshall, J., Pirie, R. (2010): Molecular and spatial epidemiology of human campylobacteriosis: source association and genotype-related risk factors. Epidemiology \& Infection, 138 (10), 1372-1383.

[13] Bessede E, Lehours P, Labadi L, Bakiri S, Megraud F. (2014): Comparison of characteristics of patients infected by Campylobacter jejuni, Campylobacter coli, and Campylobacter fetus. J Clin Microbiol 52: 328-330.

[14] Ahmed MF, Schulz J, Hartung J. (2013): Survival of Campylobacter jejuni in naturally and artificially contaminated laying hen feces. Poult Sci 92: 364-369.

[15] Ellis-Iversen J, Ridley A, Morris V, Sowa A, Harris J,
Atterbury R, Sparks N, Allen V (2012): Persistent environmental reservoirs on farms as risk factors for Campylobacter in commercial poultry. Epidemiol Infect 140: 916-924.

[16] Mungai EA, Behravesh CB, Gould LH (2015): Increased outbreaks associated with non pasteurizedmilk, United States, 2007-2012. Emerg Infect Dis 21: 119-122.

[17] Collado L, Gutierrez M, Gonzalez M, Fernandez H. (2013): Assessment of the prevalence and diversity of emergent campylobacteria in human stool sample susing a combination of traditional and molecular methods. Diagn Microbiol Infect Dis 75: 434-436.

[18] Llarena AK, Skarp-de Haan CP, Rossi M, Hanninen ML. (2015): Characterization of the Campylobacter jejuni population in the barnacle geese reservoir. Zoonoses Public Health 62: 209-221.

[19] Little CL, Gormley FJ, Rawal N, Richardson JF (2010): A recipe for disaster: outbreaks of campylobacteriosis associated with poultry liver pate in England and Wales. Epidemiol Infect 138: 1691-1694.

[20] Newell DG, Elvers KT, Dopfer D, Hansson I, Jones P, James S, Gittins J, Stern NJ, Davies R, Connerton I, Pearson D, Salvat G, Allen VM.(2011): Biosecurity-based interventions and strategies to reduce Campylobacter spp. on poultry farms. Appl Environ Microbiol 77: 8605-8614.

[21] Teh AHT, Lee SM, Dykes GA (2019): Association of some Campylobacter jejuni with Pseudomonas aeruginosa biofilms increases attachment under conditions mimicking those in the environment. PLoS One 14: e0215275.

[22] Humphrey S, Chaloner G, Kemmett K, Davidson N, Williams N, Kipar A, Humphrey T, Wigley P (2014): Campylobacter jejuni is not merely a commensal in commercial broiler chickens and affects bird welfare. Micro Bio 5: e01364-14.

[23] European Food Safety Authority (2010): Analysis of the baseline survey on the prevalence of Campylobacter in broiler batches and of Campylobacter and Salmonella on broiler carcasses in the EU, 2008, Part A: Campylobacter and Salmonella prevalence estimates. EFSA J 8: 1522.

[24] Andrew P, Emily V, Mai P, Ian Y, Nicole B (2013) Using risk factor weighting to target and create effective public health policy for Campylobacteriosis prevention in Ontario, Canada. Amer J Public Health Res 1: 32-37.

[25] Williams, L. K. et al. (2009) 'Enrichment culture for the isolation of Campylobacter spp: Effects of incubation conditions and the inclusion of blood in selective broths', International journal of food microbiology. Elsevier, 130 (2), pp. 131-134.

[26] Lake RJ, Horn BJ, Dunn AH, Parris R, Green FT, McNickle DC. (2013): Cost-effectiveness of interventions to control Campylobacter in the New Zealand poultry meat food supply. J Food Prot 76: 1161-1167.

[27] Chen J, Sun XT, Zeng Z, Yu YY. (2011): Campylobacter enteritis in adult patients with acute diarrhea from 2005 to 2009 in Beijing, China. Chin Med J (Engl) 124: 1508-1512.

[28] Beyene G and Haile-Amlak H.(2004): Antimicrobial sensitivity pattern of Campylobacter spp. among children in Jimma University Specialized Hospital, southwest Ethiopia. Ethiop J Health Dev 2004; 3: 185-189. 
[29] Kassa, T., Gebre-Selassie, S., \& Asrat, D. (2007). Antimicrobial susceptibility patterns of thermotolerant Campylobacter strains isolated from food animals in Ethiopia. Veterinary microbiology, 119 (1), 82- 87.

[30] Asrat, D., Hathaway, A., \& Ekwall, E. (1999). Studies on enteric campylobacteriosis in Tikur Anbessa and EthioSwedish children's hospital, Addis Ababa, Ethiopia. Ethiopian medical journal, 37 (2), 71-84.

[31] Dadi L, Asrat D (2008): Prevalence and antimicrobial susceptibility of thermotolerant Campylobacter strains in retail raw meat products in Ethiopia. Ethiop J Health Dev 22: 195196.

[32] Woldemariam T, Asrat D, Girma Z (2009): Prevalence of thermophilic Campylobacter spp. in carcasses from sheep and goat in Ethiopia. Ethiop J Health De 23: 229-233.
[33] Ewnetu D, Mihret A (2010): Prevalence and antimicrobial resistance of Campylobacter isolates from humans and chickens in Bahir Dar, Ethiopia. Foodborne Pathog Dis 7: 667-670.

[34] Yeshimebet C, Daniel A, Patamapom A, Winya L (2013): Prevalence and antimicrobial susceptibility of thermophilic campylobacter isolated from sheep at Debre-Birhan, NorthShoa, Ethiopia. Kasetsart J Nat Sci 47: 551-560.

[35] Abamecha A, Assebe G, Tafa B, Wondafrash B (2015): Prevalence of Thermophilic Campylobacter and their Antimicrobial Resistance Profile in Food Animals in Lare District, Nuer Zone, Gambella, Ethiopia. J Drug Res Dev 12: $1-6$. 